\title{
RELACIÓN DE LA PRESENTACIÓN DE INFORMACIÓN DE NEGOCIOS ON-LINE CON LAS VARIABLES FINANCIERAS EN LAS EMPRESAS COLOMBIANAS*
}

\author{
FABIO ENRIQUE GÓMEZ MENESES"* \& DIEGO FERNANDO CATÓLICO SEGURA*** \\ UNIVERSIDAD SANTO TOMÁS - UNIVERSIDAD CENTRAL DE BOGOTÁ
}

Recibido/Received/Recebido: 12/03/2010 - Aceptado/ Accepted/Aprovado: 26/04/2010

\begin{abstract}
Resumen
Las organizaciones hacen uso de diversos canales para la divulgación y revelación de la información de sus negocios, como por ejemplo Internet, canal a través del cual se pueden satisfacer las necesidades de los usuarios con mayor agilidad y brindando mejores condiciones de accesibilidad. La divulgación y revelación de la información puede verse determinada por el entorno y las características financieras que definen a las compañías. El presente trabajo hace un análisis de correlación entre las variables financieras y el uso de Internet para revelar y divulgar información del negocio de manera individual y según el sector económico al que pertenecen las 500 empresas más representativas de Colombia. De acuerdo con los resultados obtenidos, variables como el patrimonio, el activo y los ingresos, se diferencian significativamente en las empresas que utilizan sitios Web para revelar y divulgar la información, respecto de las que no lo hacen. En el caso del sector al que pertenecen las compañías, existen diferencias estadísticamente significativas en las variables financieras relacionadas con el tamaño de la empresa en sectores tales como: telecomunicaciones, hidrocarburos, bebidas y alimentos.
\end{abstract}

Palabras clave: Revelación de información en Internet, divulgación de información en Internet, Sitio Web, variables financieras, sector económico.

\section{RELATION BETWEEN ON-LINE BUSINESS INFORMATION PRESENTATION AND FINANCIAL VARIABLES OF COLOMBIAN COMPANIES}

\begin{abstract}
Organizations use various means to divulgate and reveal business information. Divulgation and revealing of information can be determined by the environmental and financial characteristics which define the companies. This paper analyses correlation among financial variables and use of internet to reveal and divulgate business information individually, and according to the economic sector to which belong the most representative 500 companies of Colombia. Considering the re-
\end{abstract}

\footnotetext{
El presente documento es producto del proyecto de investigación "Divulgación de reportes de negocios on-line y su relación con las variables financieras en las 500 empresas más representativas de Colombia”, el cual fue financiado por la Universidad Santo Tomás de Bogotá para los años 2009 y 2010.

* Contador Público y, Especialista en Revisoría Fiscal y Auditoría Externa - Universidad Autónoma de Bucaramanga (Colombia), Master en Administración Electrónica de Empresas, Master en Contabilidad y Finanzas y Doctorando en Contabilidad y Finanzas - Universidad de Zaragoza (España), Profesor de Tiempo Completo - Universidad Santo Tomas, Bogotá (Colombia), Grupo de investigación: contaduría: información, control e impacto social. Correo electrónico: fabiogomez@usantotomas.edu.co

** Contador Público - Universidad Nacional de Colombia, Bogotá (Colombia), Especialista en Finanzas - Universidad Santo Tomás, Bogotá (Colombia), Profesor de Tiempo Completo - Universidad Central, Bogotá (Colombia), Grupo de investigación: contaduría: información, control e impacto social. Correo electrónico: dcatolicos@ucentral.edu.co
} 
sults obtained variables such as patrimony, asset and incomes have significant differences in companies that use websites to show and publish their information in relation to those companies who does not. In the case of the sector to which the companies belong, there are statically significant differences in financial variables related to enterprise size in sectors such as: telecommunications, hydrocarbons, beverages and food.

Keywords: Information revelation, information divulgation, internet use, financial variables, economic sector.

\title{
RELAÇÃO ENTRE A APRESENTAÇÃO DE INFORMAÇÃO DE NEGÓCIOS ON-LINE E AS VARIÁVEIS FINANCEIRAS NAS FIRMAS COLOMBIANAS
}

\begin{abstract}
Resumo
As organizações utilizam diferentes canais de divulgação e informação empresarial. A disseminação da informação pode depender do ambiente e das características financeiras que definem a empresa. Neste artigo se analisa a correlação entre variáveis financeiras e uso de Internet para revelar e difundir informação individual, de acordo com o sector econômico a que pertencem as 500 firmas mais representativas na Colômbia. Conforme os resultados, as variáveis patrimônio, bens e ingressos são bastante diferentes em empresas que usam sites web para divulgar e disseminar informação e nas que não os utilizam. No caso do sector a que pertencem as empresas, houve diferenças estatisticamente significativas nas variáveis financeiras relacionadas com o porte da empresa em áreas como telecomunicações, hidrocarbonetos, alimentos e bebidas.

Palavraschave: Revelação de informação, disseminação de informação, emprego de Internet, variáveis financeiras, setor econômico
\end{abstract}

Gómez, F. \& Católico, D. (2010) Relación de la presentación de información de negocios on-line con las variables financieras en las empresas colombianas. En: Revista de la Facultad de Ciencias Económicas de la Universidad Militar Nueva Granada. rev.fac.cienc.econ, XVIII (1)

JEL: M29, M41, M49.

\section{Introducción}

En el marco de las prácticas de gobierno corporativo y, en particular, lo referente a la transparencia de la información, las organizaciones deben revelar y divulgar información que permita fundamentar la toma de decisiones gerenciales (financieras y no financieras) por parte de los diferentes grupos de interés.

Al respecto, las compañías cuentan con distintos medios para divulgar la información que se desea revelar, como son los tradicionales en medio físico o aquellos que suponen el uso de las diferentes tecnologías de la información y comunicación existentes en la actualidad, como es el caso de Internet.
No obstante, el tipo de información que se revela y los medios técnicos utilizados para divulgar dicha información, se encuentran determinados por las características externas e internas de las compañías. Entre las externas se encuentran: el sector económico, las condiciones de regulación y el entorno competitivo, y en el caso de las internas: la infraestructura tecnológica y la estructura administrativa y financiera, entre otras.

Estas variables internas han sido estudiadas a través de modelos como el TOE (Techology-OrganizationEnvironment) de Tornatzky \& Fleisher (1990) y los trabajos de Kivijarvi \& Saarinen (1995) y Osei-Bryson \& Ko (2004), en donde se analiza el peso que 
diversos factores tienen sobre la decisión de adoptar o no una innovación tecnológica. Factores como el tamaño, el sector de la economía al cual pertenezca la empresa y su situación financiera imperante, han demostrado tener una significatividad y relevancia en la decisión final de adoptar una tecnología de la información y de revelar y divulgar información financiera y no financiera a través de Internet.

Es este último factor (situación financiera) el cual será objeto de análisis en las 500 empresas más representativas de Colombia para el año 2008, en donde específicamente se estudiará la relación entre una serie de variables financieras respecto a la decisión de adoptar o no sitios Web en las empresas para la revelación y divulgación de la información.

Para ello, en el presente documento se plantea el problema objeto de investigación, lo que da origen a dos hipótesis de trabajo que exponen la posible relación entre las variables financieras de las compañías y el uso de Internet para revelar y divulgar información. Previo a la confirmación o no de las hipótesis planteadas, se desarrolló todo un referente teórico y conceptual que sustenta la problemática objeto de estudio. Finalmente, se describirán y llevarán cabo las mediciones pertinentes que permitan la validación de las hipótesis de trabajo, obteniéndose una serie de resultados que conllevarán a las conclusiones del trabajo efectuado.

\section{Planteamiento del problema e hipótesis de trabajo}

$\mathrm{Al}$ analizar los lineamientos establecidos por la normatividad contable colombiana frente a la preparación y presentación (esta última incluye la revelación y divulgación) de la información contable de las empresas, se encuentra que la información está determinada por los atributos de la organización, como lo indica el principio de características y prácticas de cada actividad: "... la contabilidad debe diseñarse teniendo en cuenta las limitaciones razonablemente impuestas por las características y prácticas de cada actividad, tales como la naturaleza de sus operaciones, su ubicación geográfica, su desarrollo social, económico y tecnológico" (LEGIS, 2010).
Estas condiciones, en especial su desarrollo económico y tecnológico, involucran sus características financieras y de infraestructura, determinando los tipos de mensajes que deben y pueden suministrar las organizaciones a los usuarios de la información, así como las formas de acceso a la información contable, es decir, se define el tipo de información que se revela y la forma como se divulga.

En cuanto al tipo de información que se revela, es de esperar que sea aquella que permita informar a los usuarios sobre aspectos que afectan a la organización o soportan la toma de decisiones. Por su parte, la divulgación reconoce que la información se comunica a los usuarios no sólo a través de los canales tradicionales, como son los documentos físicos, sino que se utilizan canales alternativos y de fácil acceso, como por ejemplo Internet (Gómez \& Católico, 2010).

De allí que se espera que las compañías dispongan de diferentes mecanismos de divulgación (Internet), para que los usuarios conozcan la información que revelan las organizaciones. Sin embargo, la utilización de este tipo de herramientas está condicionada por las percepciones y las características que definen a las empresas, como lo demuestran los modelos de aceptación de tecnologías, dentro de los cuales se puede mencionar el modelo TAM (Technology Acceptance Model) de Davis (1989) y el Modelo TOE (Technology-Organization-Environment Framework) de Tornatzky \& Fleisher (1990), los cuales exponen, en el primero de ellos, que existen una serie de percepciones (utilidad y facilidad de uso de una TIC) que determinan la adopción de una innovación tecnológica en particular, y en cuanto al segundo, se presentan una serie de entornos (tecnológico, externo y organizacional) que condicionan la decisión de adoptar una tecnología de la información y comunicación. En el entorno organizacional, se encuentra todo lo relacionado con los aspectos financieros, contables, administrativos y jurídicos que caracterizan a una organización.

Uno de los estudios en particular que evalúa la relación que tienen las variables financieras de las empresas sobre la adopción de un innovación tecnológica específica, es el de Lorca y De Andrés (2007), 
trabajo en el cual se examina la importancia de las variables financieras de una compañía frente a la adopción y utilización de sistemas ERP (Enterprise Resource Planning).

Bajo este contexto, se desea entonces, que las organizaciones adopten tecnologías que permitan la revelación y divulgación de la información del negocio para satisfacer las necesidades de información de los usuarios; no obstante, dicha adopción puede estar condicionada por las características financieras que definen a las compañías.

La divulgación de información financiera a través de Internet, mediante el uso de herramientas electrónicas, es un comportamiento que no les resulta extraño a las empresas colombianas y, por lo tanto, es importante evaluar: ¿Cuál es la relación que puede existir entre las variables financieras que caracterizan a las organizaciones, frente a la información del negocio que se revela y divulga a través de Internet?

Cuando se plantean las variables financieras que caracterizan a las empresas, se hace referencia a identificar una serie de particularidades que permitan definir, financieramente, al menos de forma parcial, a las empresas que revelan y divulgan información del negocio.

En este sentido, las variables que pueden tomarse como referencia en dicha relación, son aquellas que están presentes en la información económicofinanciera que las empresas reportan en sus estados financieros anuales y que han sido objeto de estudio en investigaciones previas como las de Poston 6 Grabski (2001), Hunton et al. (2003), Shin (2004) y Lorca \& De Andrés (2007).

De acuerdo con el planteamiento anteriormente expuesto, la presente investigación tiene las siguientes hipótesis de estudio:
H1: Existe una relación entre las variables financieras de las compañías y el uso de Internet para revelar y divulgar información del negocio de las empresas colombianas.

H2: Existe una relación entre las variables financieras de las compañías y el uso de Internet para revelar y divulgar información del negocio de las empresas colombianas de acuerdo con el sector económico al cual pertenecen.

Para desarrollar los planteamientos propuestos, se hará una revisión de los referentes teóricos y conceptuales que exponen la perspectiva desde la cual se sustenta el presente estudio, permitiendo dar soporte a las mediciones que se hacen para validar o refutar las hipótesis planteadas.

\section{El concepto de revelación y divulgación de información y su aplicación en las organizaciones}

Las organizaciones presentan información financiera y no financiera con respecto a sus operaciones, con el fin de satisfacer las necesidades de información de los grupos de usuarios que tienen algún interés en aspectos tales como, su situación financiera y desempeño financiero, entre otros.

Dentro de la información financiera y no financiera de las organizaciones se encuentra ubicada la información contable, la cual se constituye como el insumo principal para la realización de todo tipo de análisis de carácter empresarial. En consecuencia, esta información deberá ser adecuadamente revelada y divulgada entre los usuarios correspondientes. Para ello, se entiende que la revelación se soporta en el hecho de informar en forma completa todo aquello que sea necesario para comprender y evaluar correctamente la situación financiera y el desempeño de la organización ${ }^{1}$.

\footnotetext{
1 El concepto descrito se soporta en lo enunciado en el artículo 15 del Decreto 2649 de 1993, el cual puede ser consultado para profundizar al respecto.
} 
Por otra parte, la divulgación se concibe como la forma de publicar o poner a disposición la información contable para los usuarios (Gómez \& Católico, 2010), en otras palabras, sería la manera como los usuarios de la información acceden a ésta, bien sea a través de los medios físicos tradicionales (papel, CD's, prensa, etc) o por medio de canales virtuales, como por ejemplo, las páginas Web de las empresas en Internet.

La comprensión de estos dos conceptos (revelación y divulgación) debe hacerse de forma conjunta, en donde no solamente basta con que la información contable y financiera de las organizaciones este completa, si no que se requiere de adecuados mecanismos de divulgación que garanticen la accesibilidad por todos los usuarios interesados en ella.

Es importante señalar que la información que se revela y divulga dependerá de las normas y lineamientos que sobre la materia existen en los países y de los usuarios de la información al cual vaya dirigida (Galindo \& Mir, 2007). Lo anterior reconoce que habrá una información obligatoria y otra voluntaria objeto de revelación por parte de las organizaciones.

En cuanto a la obligatoriedad de la revelación de información contable en Colombia, ésta se encuentra definida principalmente en el Decreto reglamentario 2649 de 1993. De otra parte, si nos centramos en la revelación voluntaria de la información, se tienen que explorar una serie de teorías que analizan la revelación de información de las organizaciones, teniendo en cuenta las relaciones existentes entre el ente económico y diversos agentes que interactúan con ella.

- Relación accionista - Ente económico

La relación existente entre el accionista y el ente económico, que lógicamente está representado por la administración de la compañía, se puede ver claramente evidenciada en la teoría positiva de la contabilidad de Watts \& Zimmerman (1978), teoría en la cual se explora la relación que existe entre los accionistas y la administración de una empresa, y los importantes efectos que dicha relación pueda llegar a tener en los mercados de capitales. Es de esperar- se que el accionista desee que la información que publica la dirección de la empresa, no afecte negativamente el valor de su acción, por el contrario, que tal información publicada maximice su valor.

\section{- Relación inversionista - Ente económico}

Si en vez de un accionista se considera otro agente denominado "Inversor", habría que remitirse a la teoría de la "señal", de Ross (1979), teoría en la cual se hace hincapie en la confianza del inversor en el buen funcionamiento y marcha de la empresa, lo cual lógicamente, solo es posible evidenciar mediante la publicación de información financiera y no financiera por parte de la administración de la compañía.

Naturalmente a la empresa le interesa en algún momento de su existencia atraer inversores, y esta posibilidad se hará realidad dependiendo de la información que la empresa revele y publique, y de acuerdo al efecto que tal información tendrá en el mercado.

\section{- Relación competencia - Ente económico}

Uno de los agentes a los cuales la empresa debe prestar especial atención es la competencia, ya que en muchas ocasiones, de ella depende la supervivencia o no de una compañía. La teoría de los costos propietarios desarrollada en Verrecchia (1983) y Wagenhofer (1990), explica cómo la revelación de información por parte de las empresas puede beneficiar a la competencia, lo cual indudablemente originaría desventajas competitivas, que se podrían traducir en costos para la empresa, y desmotivaría a la administración de la compañía de revelar información sensible o relevante.

\section{- Relación Gobierno - Ente económico}

Como se comentó anteriormente, la primera razón por la cual una empresa revela información, es por la existencia de una normatividad que así lo exige, la cual ha sido establecida por los reguladores de un país. En este contexto se puede revisar la teoría de la regulación, en particular, su enfoque normativo, consistente en la teoría del interés publico (Arms- 
trong, Cowan \& Vickres, 1994), en donde se señala la existencia de la regulación con información asimétrica, en la cual las empresas están generalmente mejor informadas que el regulador sobre: (1) las condiciones de costos y demanda de la industria $y$, (2) sobre sus acciones, por ejemplo, su nivel de esfuerzo por reducir los costos.

Por lo tanto, el regulador necesita conocer dicha información para reglamentar ciertos hechos que afectan a las empresas, tales como, políticas fiscales (impuestos, tasas y contribuciones) o políticas de sector (concesiones, tarifas, entre otras). Lógicamente a la empresa en principio no le interesa que esto suceda, por lo cual, podría llegar a limitar o matizar la información que publique, sin que esto signifique el incumplimiento de las normas y leyes establecidas.

- Relación de otros stakeholders Ente ecónomico

La empresa se relaciona con un importante número de agentes en sus actividades normales, con estos diferentes grupos de interés las organizaciones desarrollan unos estrechos vínculos, que dado el caso, pueden afectarla o beneficiarla. La empresa en sus actuaciones, y específicamente en la revelación de información, no sólo tendrá que prestar atención en sus accionistas, sino que deberá velar por los intereses de todos aquellos agentes que se relacionen con ella, y lógicamente tendrá que velar por su propio sostenimiento.

Teniendo en cuenta este contexto, diferentes teorías, como la teoría de los stakeholders (Freeman, 1984), la teoría de la legitimación (Dowling \& Pfeffer, 1975) o la teoría institucional (Meyer \& Rowan, 1977), plantean la inclusión de información de sus actividades sociales o de información medioambiental, por citar algunos tópicos.

Según lo establecido en la teoría de los stakeholders, si la empresa mantiene una serie de vínculos estables con todos los agentes con los cuales se relaciona, su posición en el mercado será la adecuada. La estabilidad de estos "vínculos" dependerá inevitablemente de la información revelada.
Para el caso de la teoría institucional, en ocasiones los comportamientos de una compañía, y dentro de estos comportamientos se pueden citar las prácticas de revelación de información, no sólo dependen de las presiones que ejerza el mercado, sino también dependerán de las presiones del sector en el cual se encuentre inserta la empresa.

Por último, en lo que se refiere a la teoría de la legitimidad, las compañías con el ánimo de obtener el beneplácito de la sociedad en las diferentes actuaciones que realizan, pueden llegar a publicar, de forma voluntaria, información de tipo social, medioambiental, cultural, etc.

Como se puede evidenciar de las teorías planteadas anteriormente y de acuerdo con lo planteado por Verrecchia (2001), no hay una única teoría que integre todo el concepto de revelación de información, es más, se puede concluir que para las empresas resulta muy difícil dar respuesta a todos los requerimientos de información de cada uno de los agentes que con ellas interactúan, por ende, las compañías deben sopesar muy bien las ventajas y desventajas que suponen diferentes estrategias de revelación total o parcial de la información, o incluso en ocasiones, de no revelación de la información (Trombeta, 2002).

Las razones por las cuales se revela información de manera obligatoria y voluntaria, guardan relación con las características propias de las empresas y de su entorno, como son la actividad económica desarrollada, el nivel de supervisión por parte de agentes reguladores, la participación en un mercado activo de valores, la prestación de servicios públicos y el tamaño de la empresa según sus condiciones financieras, entre otras, según lo descrito en trabajos como los de Meek et al. (1995), Gallego et al. (2008) y Alonso (2009). Estos y otros trabajos abordan y estudian los conceptos de revelación de información obligatoria y voluntaria desde ambos enfoques (características de las empresas y características del entorno). En los siguientes párrafos se profundizará en algunos de ellos.

Inicialmente, si se analizan las características internas de las compañías, como por ejemplo sus con- 
Tabla 1. Ventajas y desventajas de las estrategias de revelación ${ }^{1}$

\begin{tabular}{|l|l|l|}
\hline \multicolumn{1}{|c|}{ Estrategias de revelación } & \multicolumn{1}{c|}{ Ventajas } & \multicolumn{1}{c|}{ Desventajas } \\
\hline $\begin{array}{l}\text { Revelación total } \\
\text { La empresa revela toda la información } \\
\text { que puede ser de interés para los desti- } \\
\text { natarios. }\end{array}$ & $\begin{array}{l}\text { - Máxima transparencia } \\
\text { - Buenas relaciones con los } \\
\text { inversores } \\
\text { - Bajo coste del capital }\end{array}$ & $\begin{array}{l}\text { - Sobrecarga de información } \\
\text { - Pérdida de ventaja competitiva }\end{array}$ \\
\hline $\begin{array}{l}\text { Revelación parcial } \\
\text { información que posee y oculta el resto. }\end{array}$ & $\begin{array}{l}\text { - Selectividad } \\
\text { - Difícil de distinguir desde la } \\
\text { revelación total } \\
\text { - Se evitan costes de revelación }\end{array}$ & $\begin{array}{l}\text { - Posible alto coste del capital } \\
\text { - Posible pérdida de ventaja } \\
\text { competitiva }\end{array}$ \\
\hline $\begin{array}{l}\text { Ausencia de revelación } \\
\text { La empresa no revela nada más que la } \\
\text { información que la ley le obliga a revelar. }\end{array}$ & $\begin{array}{l}\text { - Protección de la ventaja competitiva } \\
\text { - Ocultar las malas noticias }\end{array}$ & $\begin{array}{l}\text { - Alto coste del capital } \\
\text { - Mala reputación en los mercados } \\
\text {-Posible reacción de los reguladores }\end{array}$ \\
\hline
\end{tabular}

diciones financieras, estas guardan relación con los niveles de revelación de información por parte de las empresas, en dos sentidos. En el primero de ellos, se determina que de acuerdo con las características financieras de las empresas, estas revelarán en mayor o menor grado información del negocio; y en el segundo, se reconoce que la información objeto de revelación, se presenta de acuerdo con el grado de materialidad respecto al peso que tienen ciertos rubros sobre unos totales predeterminados. Esto último lo expone Hendriksen (1981), al analizar la revelación en la presentación de informes financieros, en donde se muestra que uno de los criterios tradicionales para identificar cuáles son las partidas objeto de revelación, es la relación o el peso de las distintas cuentas frente al total de activos, total ingresos o utilidades bruta o neta, que se relacionan en las cifras financieras de las empresas.

Frente a estos dos enfoques, se han desarrollado trabajos que analizan la primera relación expuesta, como es el caso de lo analizado por Meek et al. (1995), sobre 226 empresas de UK, USA y tres países continentales europeos (Alemania, Francia y Holanda), en donde se determinó que existe una influencia significativa del país o región de origen de la empresa, el tamaño de la compañía, el hecho de cotizar internacionalmente y la pertenencia a un sector económico, para determinar la presentación de información.

Posteriormente, Jaggi \& Low (2000), plantean un estudio en donde se analiza que el nivel de información de las empresas es mayor en los países con sistemas legales anglosajones que en los de corte continental. De igual forma, en este estudio se plantea que el nivel de endeudamiento incide positivamente en la presentación de información en los países anglosajones, mientras que ejerce una influencia negativa en los continentales.

Por otra parte, Gallego et al. (2008), realiza un análisis sobre las compañías más grandes que cotizan en el mercado de capitales español, en donde se evalúa la influencia de la concentración industrial, el tamaño de la compañía, el sector industrial, la rentabilidad y el endeudamiento, en la revelación obligatoria y voluntaria de la información por parte de dichas compañías.

Si bien se ha determinado la relación existente de la revelación de información y las características de las compañías y las de su entorno, cabe señalar que en estas relaciones existe un factor importante que determina la información que se presenta, el cual se sustenta en el uso de la tecnología para divulgar la información, en particular, la utilización de Internet

1 Fuente: Trombetta (2002). 
como canal para presentar la información ante los distintos Stakeholders.

Frente a lo anterior, Alonso (2009) analiza la relación de la información que las empresas difunden a través de Internet y las características que tienen las empresas más transparentes que cotizan en el mercado español. Los resultados de este trabajo evidenciaron que existe una relación positiva entre el tamaño de la empresa y su presencia en mercados extranjeros, frente a la información que se revela a través de Internet.

Otras investigaciones han analizado las relaciones existentes entre diversas variables relacionadas con la posición financiera de una empresa y la adopción de una determinada tecnología de la información y la comunicación, trabajos como los de Tornatzky \& Klein (1982), Kivijarvi \& Saarinen (1995) y Osei-Bryson \& Ko (2004) examinan indicadores tales como el tamaño, infraestructura y recursos de las compañías, su rentabilidad, endeudamiento y la productividad frente a la utilización de innovaciones tecnológicas.

Finalmente, esta relación se puede analizar desde la óptica de las administraciones públicas, citando por ejemplo el trabajo de Styles \& Tennyson (2005), en donde se encontró la existencia de una relación positiva entre la publicación de información financiera en los sitios Web de los municipios y el tamaño de los mismos. A las mismas conclusiones llegó el estudio empírico de Serrano-Cinca et al (2009), en el cual se toma una muestra de municipios españoles, donde los que revelan información financiera por Internet, son en promedio tres veces más grandes que aquellos que no revelan.

En este sentido, la revelación de información guarda relación con las características financieras de las compañías, y su percepción y disposición para adoptar ciertas tecnologías de la información, como es Internet, para efectos de su divulgación.

\section{Diseño de la investigación}

La presente investigación es de corte correlacional, dado que se analiza la relación existente entre una serie de variables financieras y la revelación y divulgación de información financiera y no financiera a través de Internet. Para ello, en los siguientes apartados se describirá la base de datos utilizada en la investigación y se presentarán y explicarán las mediciones que se efectúan para efectos de validar o refutar las hipótesis planteadas en el trabajo.

\subsection{Base de datos}

La información estadística sobre la cual se hicieron los diferentes análisis, corresponde a los datos financieros de 14 variables analizadas en las 500 empresas más grandes de Colombia, de acuerdo con sus resultados financieros para el año 2008 (Anexo 1). La información financiera de estas compañías fue tomada de publicaciones de corte empresarial realizadas en el país, principalmente de la revista cambio (www.cambio.com.co), revista que anualmente publica un ranking de las principales empresas colombianas.

Las 14 variables utilizadas correspondían a: ingresos operacionales, total activo, patrimonio, utilidad neta, margen operacional, margen neto, razón corriente, días de inventario, días de cartera, días de proveedores, endeudamiento, apalancamiento, ROA y ROE.

A partir de la información recolectada y organizada se procede a realizar todos los análisis y mediciones necesarias que permitan determinar si existe alguna relación positiva o negativa entre las variables financieras analizadas y la revelación y divulgación de información financiera y no financiera en Internet mediante un sitio Web, es decir, si tener una determinada posición financiera se constituye como un determinante estadísticamente significativo o no de la presentación de información en la Red.

\subsection{Mediciones}

Con los datos correctamente organizados en SPSS versión 15.0 (programa estadístico que se utilizará para realizar los diferentes análisis) se procede a realizar las siguientes mediciones: 
- La relación existente entre las variables financieras de las empresas y el uso de Internet mediante sitios Web para la revelación y divulgación de información.

- La relación existente entre las variables financieras de las compañías en cada uno de los sectores de la economía analizados y el uso de Internet a través de sitios Web para la presentación de información.

Para efectuar estas dos mediciones se realizaron contrastes de hipótesis con las diferentes variables de posición financiera, con el ánimo de determinar, individualmente, cuales influían en la adopción de un sitio Web por parte de las compañías analizadas para la revelación y divulgación de información.

Previó a la aplicación de los diferentes contrastes de hipótesis se verificará la normalidad de las variables utilizadas, luego se determinará el logaritmo de todas estas variables y se volverá a examinar su normalidad, aquellas que resulten ser normales, se les aplicará el test de Welch (1947) y se constrastarán sus resultados con los obtenidos en las otras técnicas de contrastes de hipótesis que se utilizarán.

Las técnicas utilizadas para la aplicación de los constrastes de hipótesis fueron el test de la ANOVA y el test no paramétrico de medias. La primera de estas técnicas, según lo descrito por Gil (2005) y siguiendo el trabajo realizado por Gómez (2010), está basada en el supuesto de que las variables que se estudian tienen una distribución normal, de no presentarse tal situación, los resultados obtenidos mediante el uso de esta prueba no serán lo suficientemente robustos.

En cuanto a la segunda técnica empleada, test no paramétricos de medias, está técnica soluciona la problemática de la normalidad en las variables, aunque también es importante acotar, que la utilización de esta prueba puede suponer la pérdida de parte de la información de las variables, ya que sus valores son cambiados a rangos, según lo explicado por Gil (2005).
La aplicación de las diferentes técnicas descritas anteriormente permitirá establecer si existe relación alguna entre las variables financieras de las compañías analizadas y la utilización de sitios Web en Internet por parte de estas compañías para la revelación y divulgación de información financiera y no financiera.

\subsection{Limitaciones en el diseño metodológico}

El presente trabajo de investigación tiene una serie de limitaciones que pueden llegar a condicionar los resultados obtenidos en las diferentes pruebas aplicadas, limitaciones que deberán ser solventadas en investigaciones posteriores que aborden la temática en cuestión.

La información financiera de las empresas sobre las cuales se realizaron los diferentes análisis, fue obtenida en un espacio determinado de tiempo y por una única vez, en consecuencia, no se pudo examinar la evolución que tales cifras tenían a lo largo del tiempo, y si la presencia en Internet mediante un sitio Web para revelar y divulgar la información financiera y no financiera, por parte de las compañías analizadas, tenía alguna incidencia o efecto positivo o negativo sobre la situación o posición financiera de las empresas consultadas.

Como segunda limitación se puede mencionar, que en esta investigación no se hizo indagación alguna acerca de las variables que favorecen u obstaculizan la adopción de sitios Web por parte de las empresas; de tal forma que no se pueden identificar claramente las razones por la cuales las compañías analizadas presentan o no su información de negocios a través de un sitio Web.

Finalmente, la última de las limitaciones hace referencia a que la base de datos analizada corresponde a empresas Colombianas de gran tamaño, dejando por fuera todas las medianas y pequeñas empresas del país, que en últimas, son la mayoría de compañías que conforman la masa empresarial en Colombia. En consecuencia, los resultados que se obtendrán en estas empresas solo serán aplicables a las grandes compañías y lógicamente no pueden ser extrapolados a organizaciones de menor tamaño. 


\section{Resultados}

Posterior a la aplicación de los diferentes análisis se obtuvieron los resultados para las dos mediciones efectuadas, la relación de las variables financieras de las empresas con la presencia o no en Internet, y la relación de las variables financieras de cada uno de los sectores de la economía analizados con la utilización o no de sitios Web por parte de las compañías, en la revelación y divulgación de información.

\subsection{Medición I}

Inicialmente fueron obtenidos los resultados respecto a la normalidad de las variables analizadas. En estos resultados se encontró que ninguna de las variables estudiadas tenía una distribución normal. Para mejorar la consistencia del análisis de normalidad, se calculó el logaritmo de las variables y se volvió a evaluar la normalidad de los datos, encontrándose en esta segunda prueba, que solo en tres de las 14 variables se cumplía la condición de normalidad (patrimonio: 0,487; utilidad neta: 0,167 y margen neto: 0,415). A estas tres variables se les aplicó el test de Welch (1947), y se encontró que sólo en una de ellas (patrimonio), existían diferencias significativas entre las compañías que tenían sitio Web en Internet y las que no lo habían implementado para efectos de revelación y divulgación. La significancia resultante en esta variable fue de 0,024.

Una vez efectuada esta primera prueba sobre las variables estudiadas, se realizaron los contrastes de hipótesis respectivos mediante la utilización de las dos técnicas descritas anteriormente (el test de la ANOVA y el test no paramétrico de medias). Con la aplicación de estas pruebas se pudo establecer la existencia de diferencias estadísticamente significativas entre un grupo y otro (las compañías que tenían página Web y las empresas que no la habían adoptado).

Para el caso de la primera de las técnicas utilizadas (ANOVA), los resultados permitieron establecer que en cinco de las 14 variables estudiadas había diferencias significativas. Estas variables correspon- dían a: los ingresos operacionales, el total del activo, el patrimonio, el margen operacional y días de inventario. Según las cifras obtenidas en cada una de estas variables, se evidenciaban diferencias estadísticamente significativas entre las empresas que tenían sitio Web, respecto de las que no lo habían implementado.

Es importante recordar, que de acuerdo con el examen de normalidad aplicado anteriormente, solo las variables de patrimonio, utilidad neta y margen neto, tenían una distribución normal, con lo cual, los resultados de esta primera técnica solo serían confiables y robustos para estas tres variables, en consecuencia, se puede aseverar, que las características de las variables y los resultados de esta primera prueba, eran significativos únicamente para la variable patrimonio, por lo tanto, se concluye de este primer examen, que el patrimonio en las empresas que tienen sitio Web, es significativamente distinto del patrimonio de las empresas que no tienen implantada esta innovación tecnológica.

En la segunda técnica aplicada (test no paramétrico de medias), los resultados son más concluyentes y se observan diferencias estadísticamente significativas en ocho de las 14 variables analizadas. Estas variables corresponden a: ingresos operacionales, total del activo, patrimonio, utilidad neta, razón corriente, días de inventario, días de cartera y endeudamiento.

Como claramente se puede observar, el 57\% de las variables financieras analizadas bajo este segundo método, presentan comportamientos lo suficientemente diferenciados entre ambos grupos, los que tienen página Web y los que no, con lo cual se puede determinar que las empresas que tienen sitio Web en Internet, tienen una situación o posición financiera diferente a la situación financiera de las empresas que no han adoptado esta innovación tecnológica. Los resultados obtenidos en cada una de las dos pruebas efectuadas (ANOVA y test no paramétricos) sobre las 14 variables analizadas son presentados en la tabla 2. 
Tabla 2. Resultados de los contrastes de hipótesis en la medición $l^{2}$

\begin{tabular}{|c|c|c|c|c|c|c|}
\hline \multirow[b]{2}{*}{ Variable } & \multirow[b]{2}{*}{ Medidas } & \multirow[b]{2}{*}{$\begin{array}{c}\text { Empresas } \\
(n=498)\end{array}$} & \multicolumn{2}{|c|}{ Tienen o no Sitio Web } & \multicolumn{2}{|c|}{ Test de medias } \\
\hline & & & $\begin{array}{l}\text { Sin Sitio Web } \\
\quad(n=150)\end{array}$ & $\begin{array}{c}\text { Con Sitio } \\
\text { Web } \\
(n=348)\end{array}$ & $\begin{array}{l}\text { ANOVA } \\
\text { (Sig.) }\end{array}$ & $\begin{array}{l}\text { No para-métricos } \\
\text { (Sig.) }\end{array}$ \\
\hline \multirow{2}{*}{ Ing_Ope } & Media & $5,0 \mathrm{E}+011$ & $2,9 \mathrm{E}+011$ & $6,0 \mathrm{E}+011$ & \multirow[b]{2}{*}{$(0,000)$} & \multirow[b]{2}{*}{$(0,000)$} \\
\hline & Mediana & $2,5 \mathrm{E}+011$ & $2,1 \mathrm{E}+011$ & $2,7 \mathrm{E}+011$ & & \\
\hline \multirow{2}{*}{ Tot_Act } & Media & $7,28 \mathrm{E}+011$ & $4,08 \mathrm{E}+011$ & $8,66 \mathrm{E}+011$ & \multirow[b]{2}{*}{$(0,012)$} & \multirow[b]{2}{*}{$(0,000)$} \\
\hline & Mediana & $1,98 \mathrm{E}+011$ & $1,34 \mathrm{E}+011$ & $2,41 \mathrm{E}+011$ & & \\
\hline \multirow{2}{*}{ Patrim } & Media & $4,19 \mathrm{E}+011$ & $2,28 \mathrm{E}+011$ & $5,01 \mathrm{E}+011$ & \multirow[b]{2}{*}{$(0,024)$} & \multirow[b]{2}{*}{$(0,000)$} \\
\hline & Mediana & 9753100000 & 72865500000 & $1,20 \mathrm{E}+011$ & & \\
\hline \multirow{2}{*}{ Util_Neta } & Media & $4,3 \mathrm{E}+010$ & $3,2 \mathrm{E}+010$ & $4,8 \mathrm{E}+010$ & \multirow[b]{2}{*}{$(0,563)$} & \multirow[b]{2}{*}{$(0,003)$} \\
\hline & Mediana & $1,0 \mathrm{E}+010$ & $5,6 \mathrm{E}+009$ & $1,1 \mathrm{E}+010$ & & \\
\hline \multirow{2}{*}{ Mar_Ope } & Media & 9,509 & 12,424 & 8,249 & \multirow[b]{2}{*}{$(0,038)$} & \multirow[b]{2}{*}{$(0,359)$} \\
\hline & Mediana & 5,900 & 5,000 & 6,300 & & \\
\hline \multirow{2}{*}{ Mar_Neto } & Media & 6,762 & 8,501 & 6,013 & \multirow[b]{2}{*}{$(0,192)$} & \multirow[b]{2}{*}{$(0,169)$} \\
\hline & Mediana & 3,200 & 2,350 & 3,400 & & \\
\hline \multirow{2}{*}{ Raz_Cor } & Media & 1,766 & 1,546 & 1,861 & \multirow[b]{2}{*}{$(0,196)$} & \multirow[b]{2}{*}{$(0,004)$} \\
\hline & Mediana & 1,300 & 1,200 & 1,400 & & \\
\hline \multirow{2}{*}{ Días_Inv } & Media & 42,725 & 36,023 & 45,730 & \multirow[b]{2}{*}{$(0,035)$} & \multirow[b]{2}{*}{$(0,047)$} \\
\hline & Mediana & 34,800 & 29,900 & 36,500 & & \\
\hline \multirow{2}{*}{ Días_Car } & Media & 52,360 & 46,256 & 55,089 & \multirow[b]{2}{*}{$(0,216)$} & \\
\hline & Mediana & 42,200 & 28,050 & 46,800 & & $(0,000)$ \\
\hline חíac Pro & Media & 32,901 & 32,696 & 32,992 & & \\
\hline Das_tu & Mediana & 24,000 & 24,000 & 24,000 & $(0,934)$ & $(0,785)$ \\
\hline Endeud & Media & 82,666 & 55,595 & 94,334 & & \\
\hline Endeud & Mediana & 47,750 & 53,300 & 46,750 & $(0,467)$ & $(0,012)$ \\
\hline Analan & Media & 28,772 & 78,709 & 7,247 & & \\
\hline Aparan & Mediana & 1,000 & 1,100 & 0,900 & $(0,459)$ & $(0,063)$ \\
\hline & Media & 0,134 & 8,613 & $-3,520$ & & \\
\hline ROA & Mediana & 4,400 & 4,250 & 4,500 & $(0,160)$ & $(0,938)$ \\
\hline $\mathrm{BOF}$ & Media & 10,903 & 10,508 & 11,073 & & \\
\hline RUE & Mediana & 10,100 & 10,750 & 9,600 & $(0,980)$ & $(0,384)$ \\
\hline
\end{tabular}

\footnotetext{
2 Fuente: Elaboración propia.
} 


\subsection{Medición II}

Respecto a la segunda de las mediciones, los resultados obtenidos en la primera de las dos técnicas (ANOVA), permitieron establecer diferencias significativas en las variables de siete de los 36 sectores estudiados (Telecomunicaciones, bebidas, minería, alimentación, productos plásticos, producción de papel y cartón y otras actividades).

Debido a las limitaciones que ofrece está técnica, las cuales fueron comentadas en apartados anteriores, se aplicó el test no paramétrico de medias, el cual nuevamente evidenció diferencias significativas en las variables de tan sólo ocho de los 36 sectores evaluados (hidrocarburos, telecomunicaciones, combustibles, bebidas, alimentación, siderúrgicas, químicos y otras actividades).

En este segundo análisis, se mantienen los sectores de telecomunicaciones, bebidas, alimentación y otras actividades respecto del primer examen efectuado, $y$ aparecen como adicionales los sectores de hidrocarburos, combustibles, siderúrgicas y químicos.
Las variables que evidenciaron comportamientos significativos en cada uno de estos ocho sectores, corresponden a: ingresos operacionales, total activo, patrimonio, utilidad neta, endeudamiento, margen operacional, margen neto, ROA, razón corriente, apalancamiento y días de inventario.

Los resultados obtenidos en cada una de las dos pruebas efectuadas (ANOVA y test no paramétrico) sobre las 14 variables analizadas en los ocho sectores descritos anteriormente, son presentados en las tablas 3 y 4.

De acuerdo con los resultados obtenidos en la segunda medición, mediante la utilización de la técnica "test no paramétrico de medias", se pudo establecer que en ocho sectores, que representan el $22 \%$ del total de sectores de la economía analizados, existe una relación entre las variables financieras y la revelación y divulgación de información a través de Internet mediante sitios Web. En los 28 sectores restantes resulta irrelevante dicha relación.

Tabla 3. Resultados de los contrastes de hipótesis (ANOVA) en la medición II ${ }^{3}$

\begin{tabular}{|l|l|c|}
\hline \multirow{2}{*}{ SECTOR } & \multicolumn{2}{c|}{ TEST ANOVA } \\
\cline { 2 - 3 } & \multicolumn{1}{|c|}{ Variable } & Significatividad \\
\hline \multirow{3}{*}{ Telecomunicaciones } & - Total Activo de la empresa & $(0,031)$ \\
& $\begin{array}{l}\text { - Patrimonio de la empresa } \\
\text { - Margen operacional }\end{array}$ & $(0,028)$ \\
\hline Bebidas & - Margen operacional & $(0,049)$ \\
\hline Minería & - Ingresos operacionales & $(0,003)$ \\
\hline \multirow{3}{*}{ Alimentación } & - Ingresos operacionales & $(0,029)$ \\
\hline Productos Plásticos & - Endeudamiento & $(0,011)$ \\
\hline Producción de papel y cartón & - ROA & $(0,029)$ \\
\hline Otras actividades & - Apalancamiento & $(0,026)$ \\
\hline
\end{tabular}

3 Fuente: Elaboración propia. 
Tabla 4. Resultados de los contrastes de hipótesis (test no paramétricos) en la medición II

\begin{tabular}{|l|l|c|}
\hline \multirow{2}{*}{ SECTOR } & \multicolumn{2}{|c|}{ TEST NO PARAMÉTRICOS } \\
\cline { 2 - 3 } & \multicolumn{1}{|c|}{ Variable } & Significatividad \\
\hline \multirow{2}{*}{ Hidrocarburos } & - Ingresos operacionales & $(0,011)$ \\
& - Total Activo de la empresa & $(0,034)$ \\
\hline \multirow{2}{*}{ Telecomunicaciones } & - Total Activo de la empresa & $(0,027)$ \\
& - Patrimonio de la empresa & $(0,011)$ \\
\hline Combustibles & - Margen operacional & $(0,038)$ \\
\hline \multirow{2}{*}{ Bebidas } & - Margen operacional & $(0,021)$ \\
& - Razón Corriente & $(0,028)$ \\
\hline \multirow{5}{*}{ Alimentación } & - Ingresos operacionales & $(0,017)$ \\
& - Total Activo de la empresa & $(0,003)$ \\
& - Patrimonio de la empresa & $(0,000)$ \\
& - Utilidad neta de la empresa & $(0,000)$ \\
& - Margen neto de la empresa & $(0,003)$ \\
\hline Siderúrgicas & - Endeudamiento & $(0,020)$ \\
\hline Químicos & - ROA & $(0,033)$ \\
\hline Otras actividades & - Ingresos operacionales & $(0,033)$ \\
\hline
\end{tabular}

\section{Conclusiones}

En esta investigación se ha estudiado si el comportamiento financiero de una serie de empresas, se relaciona de forma significativa con la presencia en Internet de estas compañías mediante un sitio Web para la revelación y divulgación de información. Para lograr tal objetivo, se tomó la información financiera de 14 variables en las 500 empresas más grandes de Colombia, y sobre tales cifras se evaluó si una determinada posición o situación financiera en las compañías analizadas, se constituía como un determinante estadísticamente significativo de su presencia en Internet. Dicho análisis también se realizó teniendo en cuenta el sector económico al cual pertenecían cada una de las compañías estudiadas.

Los resultados obtenidos en la primera de las mediciones permitieron evidenciar, para el caso de la primera de las técnicas aplicadas (Test de la ANO-
VA), que solo en la variable de patrimonio existían diferencias estadísticamente significativas entre el grupo de las que tenían sitio Web y el grupo de las que no. Para el caso de la segunda prueba (test no paramétricos), ocho de las 14 variables analizadas arrojaban diferencias estadísticamente significativas entre el grupo de las que tienen sitio Web y las que no utilizan esta herramienta.

Estos resultados permiten llegar a la conclusión que la posición o situación financiera de las empresas que tienen presencia en Internet mediante un sitio Web es significativamente diferente de las compañías que no hacen uso de esta tecnología de la información, encontrándose que en las empresas que si tienen página en Internet, sus ingresos operacionales, su activo total, su patrimonio, su utilidad neta y su nivel de endeudamiento son significativamente superiores que en las empresas que no adoptan dicha tecnología. Es importante notar que tanto en la primera como en la segunda de las pruebas

\footnotetext{
4 Fuente: Elaboración propia.
} 
aplicadas, el patrimonio aparece como una variable determinante en la revelación y divulgación de información, confirmando así su relevancia dentro del análisis efectuado.

Todas estas variables son generalmente indicativas del tamaño de una organización, con lo cual se puede concluir que las empresas que adoptan y usan sitios Web son compañías de mayor tamaño respecto de aquellas que no los usan. En consecuencia, el tamaño de una empresa es un importante determinante en la adopción y uso de tecnologías de la información y comunicación, conclusión que es confirmada y soportada por múltiples trabajos que evalúan esta variable, como por ejemplo, los de Tornatzky \& Klein (1982), Styles \& Tennyson (2005) y Serrano-Cinca et al (2009).

Resulta de gran importancia destacar que autores como Lee \& Xia (2006) ponen en duda la existencia de esta relación, indicando que los resultados empíricos obtenidos en diversos estudios han sido mixtos e inconsistentes, principalmente debido a la existencia de una serie de factores que matizan dicha relación, factores tales como el tipo de innovación tecnológica que se adopta, el sector, el tipo de organización, la fase o etapa en la cual se encuentra la innovación, el ámbito y el tipo de medida utilizado en la variable tamaño.

En cuanto a las variables razón corriente, días de inventario y días de cartera, los resultados de estos indicadores en las empresas que adoptaron esta tecnología, eran superiores a las cifras obtenidas por las empresas que no adoptan sitios Web para la revelación y divulgación de información financiera y no financiera. De acuerdo con estos resultados, se puede establecer que las empresas que tienen sitio Web, tienen una mejor capacidad de pago que las que no adoptan esta innovación, pero el periodo de tiempo en que rota el inventario y la cartera de estas empresas, es inferior al de las compañías que implementan esta tecnología de la información y la comunicación.

Respecto a la segunda de las mediciones, que buscaba determinar si existían diferencias significativas en las variables financieras en cada uno de los secto- res analizados, los resultados permitieron establecer que de los 36 sectores económicos estudiados, solo en ocho de ellos se presentaban diferencias estadísticamente significativas en algunas de las variables estudiadas de las empresas que adoptaron esta tecnología respecto de aquellas que no lo habían hecho.

Estos ocho sectores aparecieron en la segunda de las pruebas aplicadas (test no paramétricos) y corresponden a: hidrocarburos, telecomunicaciones, combustibles, bebidas, alimentación, siderúrgicas, químicos y otras actividades. Resulta importante destacar que dentro de las variables que resultaron ser estadísticamente significativas en cada uno de estos sectores, se encuentran todas aquellas relacionadas con el tamaño de la empresa, como por ejemplo, los ingresos operacionales, el total del activo, el patrimonio y la utilidad, entre otras, lo cual ratifica la importancia del tamaño de la organización como determinante en la adopción de innovaciones tecnológicas, tal cual como fue comentado en las conclusiones de la primera de las mediciones.

Finalmente, si bien es cierto que los análisis demostraron que el tamaño resulta ser una variable importante que puede llegar a limitar la divulgación y revelación de información de negocios de una empresa en Internet a través de una página Web, en nuestra opinión, el tener presencia en Internet es una ventaja significativa que le puede brindar a las organizaciones importantes beneficios, y en donde las inversiones que se requieren para implementar esta innovación son relativamente bajas y menos costosas que las utilizadas tradicionalmente.

\section{Futuras líneas de investigación}

De acuerdo con los resultados obtenidos y teniendo en cuenta las limitaciones expuestas en el diseño metodológico en la presente investigación, resulta pertinente profundizar las temáticas analizadas mediante futuras investigaciones que abarquen y soluciones las limitaciones planteadas.

Específicamente se propone la continuación de este estudio mediante una investigación que permita examinar como la presencia en Internet mediante 


\section{RELACIÓN DE LA PRESENTACIÓN DE INFORMACIÓN DE NEGOCIOS ON-LINE CON LAS VARIABLES FINANCIERAS EN LAS EMPRESAS COLOMBIANAS}

un sitio Web para revelar y divulgar la información financiera y no financiera, puede tener alguna incidencia o efecto positivo o negativo sobre la situación o posición financiera de las empresas consultadas.

Igualmente, resultaría fundamental investigar sobre aquellos factores que favorecen la adopción de esta innovación tecnológica, de tal forma que las compañías pudieran "apuntarle" a la consecución de tales factores, y en cuanto a las variables que inhiben a las empresas en la adopción de una página en Internet, su identificación permitiría su posterior eliminación o solución.

Por último, sería de gran utilidad realizar una nueva investigación, donde se incluyan empresas de todos los tamaños, de tal forma que se puede presentar un completo panorama de las compañías Colombianas, acerca de su presencia en Internet mediante sitios Web y de la información que en ellos se revela y divulga.

\section{Referencias}

Armstrong, M., Cowan, S. \& Vickers, J. (1994). Regulatory Reform. Economic Analysis and British Experience. MIT Press, Cambridge, Mass. p. 27.

Alonso, M. (2009). La transparencia de las empresas en internet para la confianza de los accionistas e inversores: un análisis empírico. En: Revista Cuadernos de Administración, Pontificia Universidad Javeriana, Vol. 22, 38: 11-30.

Davis, F. D. (1989). Perceived usefulness, perceived ease of use and user acceptance of information technology. MIS Quarterly, 13 (3), 319-339.

Dowling, J. \& Pfeffer, J. (1975). Organization legitimacy: social values and organizational behavior. Pacific Sociological Review, (18:1), $122-136$.

Freeman, E. R. (1984). Strategic Management: a Stakeholder Approach, Pitman Publishing.

Galindo, R. \& Mir, C. (2007). Gobierno Corporativo, Transparencia y Auditoria. Revista Internacional LEGIS de Contabilidad \& Auditoria, (30), 147-170.

Gallego, I., García, I. \& Rodríguez, L. (2008). Voluntary and compulsory information disclosed online: the effect of industry concentration and other explanatory factors. En: Online Information Review, Emerald Group Publishing Limited, Vol. 32, 5: 596-622

Gil, J. (2005). Aplicación del método Bootstrap al contraste de hipótesis en la investigación educativa. Revista de Educación, 336, pp. 251-265

Gómez, F. (2010). La factura electrónica en las empresas aragonesas: perfil financiero, tecnológico y efectos de la implantación. Tesis Doctoral, Departamento de Contabilidad y Finanzas, Universidad de Zaragoza, Aragón, España.
Gomez, F. \& Católico, D. (2010). Revelación y divulgación de la información financiera y no financiera on-line de las 500 empresas más representativas en Colombia. Revista Cuadernos de Contabilidad, 27. Documento aprobado y pendiente de publicación.

Hunton, J. E., Lippincott, B. \& Reck, J. L. (2003). Enterprise resource planning systems: comparing firm performance of adopters and non adopters. International Journal of Accounting Information Systems, 4, pp. 165-184.

Hendriksen, E. (1981). Teoría de la contabilidad. Ciudad de México: Unión Tipográfica Editorial.

Jaggi, B. \& Low, P. (2000). Impact of culture, market forces, and legal system on financial disclosures. En: The International Journal of Accounting, Vol. 35, 4, pp. 495-519.

Kivijarvi, H. \& Saarinen, T. (1995). Investment in information systems and the financial performance of the firm. Information \& Management, 28 pp. 143-163.

Lee, G. \& Xia, W. (2006). Organizational size and IT innovation adoption: A meta -analysis. Information \& Management 43, pp. 975-985.

LEGIS (2010). PUC, Bogotá: Legis Editores S.A.

Lorca, P. \& De Andrés, J. (2007). Efectos de la implantación de sistemas integrados de gestión (ERP) en las grandes empresas españolas. Revista Española de Financiación y Contabilidad. 36 (135), pp. 595-623.

Meek, G., Roberts, C. B. \& Gray, S. (1995). Factors influencing voluntary annual report disclosures by US, UK and Continental European multinational corporations. En: Journal of International Business Studies. 3rd quarter, pp. 555-572.

Meyer, J. W. \& Rowan, B. (1977). Institutionalized organizations: formal structure as myth and ceremony. American Journal of Sociology, (8), 340-363.

Osei-Bryson, K.M. \& Ko, M. (2004). Exploring the relationship between information technology investments and firm performance using regression splines analysis. Information \& Management, 42, pp. 1-13.

Poston, R. \& Grabski, S. (2001). Financial impacts of enterprise resource planning implementations. International Journal of Accounting Information Systems, 2 (4), pp. 271-294.

Ross, S. A. (1979). Disclosure regulation in the financial markets: implications of modern finance theory and signalling theory. Issues in Financial Regulation, New York. McGraw-Hill.

Serrano-Cinca C., Rueda-Tomás M. \& Portillo-Tarragona, P. (2009). Factors influencing e-disclosure in local public administrations. Environment and Planning C: Government and Policy, 27 (2), pp. $355-378$.

Shin, N. (2004). The impact of information technology on the financial performance of diversified firms. Decision Support Systems, 41, pp. 698-707.

Styles, A. \& Tennyson, M. (2005). The Accessibility of Financial Reporting of U.S. Municipalities on the Internet. Paper presented at American Accounting Association Mid-Atlantic Regional Meeting, Philadelphia, PA.

Tornatzky, L.G. \& Fleischer, M. (1990). The Processes of Technological Innovation. Lexington Books, Lexington, Massachusetts.

Tornatzky, L.G. \& Klein, K.J. (1982). Innovation characteristics and innovation adoption-implementation: a meta-analysis of findings. IEEE Transactions on Engineering Management, 29, pp. $28-45$. 
Trombetta, M. (2002). La decisión de revelar voluntariamente información de la empresa: desde el análisis teórico a una propuesta metodológica. Documento de Trabajo. Departamento de Economía de la Empresa. Universidad Carlos III. Madrid.

Verrecchia, R. E. (1983). Discretionary Disclosure. Journal of Accounting and Economics, (5), 179-194.

Verrecchia, R. E. (2001). Essays on disclosure. Journal of Accounting and Economics, 32 (1-3), 97-180.
Wagenhofer, A. (1990). Voluntary disclosure with a strategic opponent. Journal of Accounting and Economics, 12, (4), 341 363.

Watts, R. L. \& Zimmerman, J. L. (1978). Towards a positive theory of the determination of accounting standards. Accounting Review, (53), 112-134.

Welch, B. L. (1947). The generalization of "Student's" problem when several different population variances are involved, Biometrika 34 (1-2). 
Anexo 1.

\section{Empresas Colombianas objeto de estudio}

\begin{tabular}{|c|c|c|c|c|c|}
\hline No. & EMPRESA & No. & EMPRESA & No. & EMPRESA \\
\hline 1 & Ecopetrol & 51 & Diaco & 101 & Schlumberger Surenco \\
\hline 2 & Comcel & 52 & Alpina & 102 & Ferrasa \\
\hline 3 & Terpel & 53 & Caja Ret. Ff.mm. & 103 & Manufacturas Eliot \\
\hline 4 & Exxon Mobil & 54 & Colgate Palmolive & 104 & Enka \\
\hline 5 & Almacenes Éxito & 55 & Nacional De Chocolates & 105 & Amov Colombia \\
\hline 6 & Telefónica & 56 & Petroquímica Colombiana & 106 & Electrificadora De Santander \\
\hline 7 & Gm Colmotores & 57 & Iss & 107 & Colombina \\
\hline 8 & Bavaria & 58 & Centelsa & 108 & Makro \\
\hline 9 & Epm & 59 & Universidad Nacional & 109 & Bp Santiago \\
\hline 10 & Avianca & 60 & Monómeros & 110 & Famisanar Eps \\
\hline 11 & Carrefour & 61 & Compensar & 111 & Contegral Medellín \\
\hline 12 & Chevron Texaco & 62 & Petrobras & 112 & Jhonson \& Jhonson \\
\hline 13 & Sofasa & 63 & Gas Natural & 113 & Sánitas Eps \\
\hline 14 & Drummond & 64 & Colcerámica & 114 & Comfernalco Antioquia \\
\hline 15 & Carulla Vivero & 65 & Isa & 115 & Zenú \\
\hline 16 & Codensa & 66 & Bel Star & 116 & Acerias Paz Del Rio \\
\hline 17 & Supertiendas Olímpica & 67 & Hewlett Packard & 117 & Rcn Televisión \\
\hline 18 & Carbones Del Cerrejon & 68 & Colombia Móvil & 118 & Susalud Eps \\
\hline 19 & Colombiana De Comercio & 69 & Samsung & 119 & Fábrica Nacional De Autopartes \\
\hline 20 & Electricaribe & 70 & Colpapel & 120 & Casa Luker \\
\hline 21 & Bp Exploration & 71 & Epsa & 121 & Molinos Roa \\
\hline 22 & Cementos Del Caribe & 72 & Grupo Aval & 122 & Valle Del Cauca - Comfandi \\
\hline 23 & Saludcoop & 73 & Cartón De Colombia & 123 & Mercedez Benz \\
\hline 24 & Etp & 74 & Gecolsa & 124 & Baxter \\
\hline 25 & $\mathrm{Hocol}$ & 75 & Lg Electronics & 125 & Colsánitas Prepagada \\
\hline 26 & Cerromatoso & 76 & Postobón & 126 & Quala \\
\hline 27 & Cca & 77 & Shell Combustibles & 127 & Casa Editorial El Tiempo \\
\hline 28 & Coomeva Eps & 78 & Tecnoquímicas & 128 & De Antioquia Comfama \\
\hline 29 & Emgesa & 79 & Avon Colombia & 129 & Didacol \\
\hline 30 & Cerrejón & 80 & Copidrogas & 130 & Pintuco \\
\hline 31 & Colanta & 81 & Fabricato & 131 & Aes Chivor \\
\hline 32 & Propilco & 82 & Cervercería Union & 132 & Los Coches \\
\hline 33 & Emcali & 83 & Brío & 133 & Holcim \\
\hline 34 & Colsubsidio & 84 & Salud Total Eps & 134 & Goodyear \\
\hline 35 & Fondo Nacional Del Café & 85 & Tepma & 135 & Acesco \\
\hline 36 & Oxycol & 86 & Siemens & 136 & Industrias Del Maíz \\
\hline 37 & Casur Policía Nacional & 87 & Productos Familia & 137 & Caracol Tv \\
\hline 38 & Cafam & 88 & Procter \& Gamble & 138 & Dell Colombia \\
\hline 39 & Epm Telecomunicaciones & 89 & Genercom Del Caribe S.a. & 139 & Autotécnica Colombiana \\
\hline 40 & Cemex & 90 & Galletas Noél & 140 & Omimex \\
\hline 41 & Eaab & 91 & Mabe & 141 & Universidad De Antioquia \\
\hline 42 & Isagen & 92 & Copservir & 142 & Ci Mundo Metal \\
\hline 43 & Sodimac & 93 & Carbone Rodríguez & 143 & Incauca \\
\hline 44 & Prodeco & 94 & Peldar & 144 & Ciamsa \\
\hline 45 & Oxy Andina & 95 & Central De Inversiones & 145 & Sony Corporation \\
\hline 46 & Nestlé & 96 & Rica Rondo & 146 & Halliburton \\
\hline 47 & Panamco & 97 & Dinissan & 147 & Avidesa Mac Pollo \\
\hline 48 & Hyundai & 98 & Riopaila Industrial S.a. & 148 & Dow Química \\
\hline 49 & Carcafé & 99 & Cafesalud Eps & 149 & Metrokía \\
\hline 50 & Cacharrería La 14 & 100 & Propal & 150 & Haceb \\
\hline
\end{tabular}




\begin{tabular}{|c|c|c|c|c|c|}
\hline No. & Empresa & No. & Empresa & No. & Empresa \\
\hline 151 & G Y J Ferreterias & 201 & Prod. Naturales De La Sabana & 251 & Gensa S.a. \\
\hline 152 & Manuelita & 202 & Sos Eps & 252 & Audifarma \\
\hline 153 & Valorem & 203 & Racafé & 253 & Aaa \\
\hline 154 & Frito Lay & 204 & Dow Agrosciences & 254 & Energía Empresarial de la Costa \\
\hline 155 & Epsifarma & 205 & Gases De Occidente & 255 & Farmasánitas \\
\hline 156 & Suzuki Motor & 206 & Coomeva Emp & 256 & Petrominerales Colombia Ltd \\
\hline 157 & Suramericana Seguridad Social & 207 & Finca & 257 & Andercol \\
\hline 158 & Solsalud Eps & 208 & Ci Yumbo & 258 & Continental Automotora \\
\hline 159 & Distoyota & 209 & Cadbury Adams & 259 & Familia Del Pacífico \\
\hline 160 & Oleoducto Central & 210 & Coopcafé Café & 260 & Pimpollo \\
\hline 161 & Coltabaco & 211 & Energía De Cundinamarca & 261 & Fundición Escobar \\
\hline 162 & Indumil & 212 & Eficacia Subcont. & 262 & Procaps \\
\hline 163 & Icollantas & 213 & Tabacalera De Colombia & 263 & Autoniza \\
\hline 164 & Compensar Eps & 214 & Ci Petróleos Del Milenio & 264 & Perenco \\
\hline 165 & Automarket & 215 & Comfenalco Valle & 265 & Pfizer \\
\hline 166 & Ford Motor & 216 & Coltejer & 266 & Embotelladora Román \\
\hline 167 & Abocol & 217 & Aventis Pharma & 267 & Parmalat \\
\hline 168 & Banacol & 218 & Comfenalco Ant Eps & 268 & Cafeagraria \\
\hline 169 & Roche & 219 & Ci Acepalma & 269 & Daity Partnermericas \\
\hline 170 & Sidunor & 220 & Cruz Blanca Eps & 270 & Grasas \\
\hline 171 & Cellstar De Colombia & 221 & Ci J. Gutiérrez Y Cía. & 271 & Brenntag \\
\hline 172 & Chec & 222 & Shell & 272 & Papeles Nacionales \\
\hline 173 & Transgas Del Interior & 223 & Acción & 273 & Sucromiles \\
\hline 174 & Tubos Del Caribe & 224 & Cent. Eléctricas Norte Santander & 274 & Motores Y Máquinas \\
\hline 175 & Abbott Laboratorios & 225 & Cacharrería Mundial & 275 & Universidad Del Valle Cauca \\
\hline 176 & Metroconcreto & 226 & Sidenal & 276 & Energética Del Tolima \\
\hline 177 & Skn Caribecafé & 227 & Ingenio Mayaguez & 277 & Refisal \\
\hline 178 & Fondo Ferrocarriles Nals. & 228 & Suramericana De Inversiones & 278 & Autolarte \\
\hline 179 & Conconcreto & 229 & Nexen Petroleum & 279 & Internacional De Vehículos \\
\hline 180 & Gases Del Caribe & 230 & Humana Vivir Eps & 280 & Distracom \\
\hline 181 & Gaseosas Lux & 231 & Meals De Colombia & 281 & Satena \\
\hline 182 & Serrano Gomez & 232 & Mps Mayorista & 282 & Levapán \\
\hline 183 & Harinera Del Valle & 233 & Detergentes & 283 & Mac \\
\hline 184 & Unilever Andina & 234 & Acegrasas & 284 & John Restrepo \\
\hline 185 & Colcafé & 235 & Vestimundo & 285 & Dispapeles \\
\hline 186 & Bayer & 236 & Italcol De Occidente & 286 & Schering - Plough \\
\hline 187 & Incolmotos Yamaha & 237 & Leonisa & 287 & Dupont \\
\hline 188 & Biofilm Prod. & 238 & Css Constructores & 288 & Yara \\
\hline 189 & Ci Equipos Y Maquinaria & 239 & Florhuila & 289 & Impresistem \\
\hline 190 & Orbitel & 240 & Novartis & 290 & Altipal Bogotá \\
\hline 191 & $\mathrm{lbm}$ & 241 & Clínicas Colsánitas & 291 & Alianza Mayorista S.a. \\
\hline 192 & Bayer Cropscience & 242 & Coéxito & 292 & Etaservicios \\
\hline 193 & Hupelcol & 243 & Fesa & 293 & Pavco \\
\hline 194 & Servientrega & 244 & E2 Energía Eficiente & 294 & Whirlpool Colombia \\
\hline 195 & Yanbal & 245 & Panamericana Librería & 295 & Meta Petroleum \\
\hline 196 & Alfagrés & 246 & Glaxosmithkline & 296 & Cafeunión \\
\hline 197 & Pride Colombia Services & 247 & Inv. Nacional De Chocolates & 297 & Organización Solarte \\
\hline 198 & Conalvías & 248 & Arroz Diana & 298 & Colmédica Eps \\
\hline 199 & Gasesosas Colombianas & 249 & Productora De Cables & 299 & Activos \\
\hline 200 & Ingenio Providencia & 250 & Empresa De Energía De Boyacá & 300 & Aluminio Nacional \\
\hline
\end{tabular}




\begin{tabular}{|c|c|c|c|c|c|}
\hline No. & Empresa & No. & Empresa & No. & Empresa \\
\hline 301 & Trilladora Union & 351 & Publicar & 401 & Camaguey \\
\hline 302 & Ci Colombiana De Carbón & 352 & Carvajal & 402 & Bimbo De Colombia \\
\hline 303 & Corpacero & 353 & Ci Inversiones Guti & 403 & Gtech Colombia Ltda. \\
\hline 304 & Ci Agrícola De Santander & 354 & Fagrave & 404 & Construcciones Colpatria \\
\hline 305 & Ci Uniban & 355 & Mercantil Colpatria & 405 & Nalsani \\
\hline 306 & Ind. De Ejes Y Transmiiones & 356 & Boehringer Ingelheim & 406 & Panamericana Formas E Impresos \\
\hline 307 & C.i Vanoil & 357 & Alimentos Friko & 407 & Sika Colombia \\
\hline 308 & Sodexho Colombia & 358 & Challenger & 408 & Rodríguez Franco \\
\hline 309 & Ecofértil & 359 & Ci Mineros Exportadores & 409 & Transgás De Occidente \\
\hline 310 & Coomeva & 360 & C.i. Braytex S.a. & 410 & Grasas Y Aceites Vegetales \\
\hline 311 & Cafihuila Ltda. & 361 & Carboquímica & 411 & Procesadora De Arroz \\
\hline 312 & Panasonic De Colombia & 362 & Unión Eléctrica & 412 & Constructora Bolívar Bogotá \\
\hline 313 & Protela & 363 & Arcesa & 413 & Distribuidora Avícola \\
\hline 314 & Yazaki Ciemel & 364 & Coonalcafé & 414 & Transselca \\
\hline 315 & Comertex & 365 & Meriléctrica & 415 & Proleche \\
\hline 316 & Telmex Colombia & 366 & Metalúrgicas & 416 & Districolombina \\
\hline 317 & Permoda & 367 & Cedenar & 417 & Sumicol \\
\hline 318 & Fajobe & 368 & Weatherford & 418 & Almacenes Yep \\
\hline 319 & Laboratorio Lafrancol & 369 & Aires & 419 & Empresa Urrá \\
\hline 320 & Yazaki Ciemel Ftz & 370 & Comfamiliar Del Huila & 420 & Didetexco \\
\hline 321 & Whitewater Inversiones & 371 & Electrohuila & 421 & Masering \\
\hline 322 & Pizano & 372 & Sanautos & 422 & Plastilene \\
\hline 323 & Distribuciones Axa & 373 & Gas Natural Comprimido & 423 & Ci Fundición Ramírez \\
\hline 324 & Henkel Colombiana & 374 & Sed International & 424 & Intermunicipal $\mathrm{Cba}$ \\
\hline 325 & Flexo Spring & 375 & Cusezar & 425 & Frigoríficos Ganaderos De Col. \\
\hline 326 & Laboratorios Wyeth & 376 & Hojalata Y Laminados & 426 & Ingenio Risaralda \\
\hline 327 & Aga Fano & 377 & Lafayette & 427 & Universidad Cooperativa \\
\hline 328 & Mercados De Familia & 378 & Chaneme Comercial & 428 & Ircc Restaurantes Casuales \\
\hline 329 & Grasas Y Productos Químicos & 379 & Calcetines Crystal & 429 & Marval \\
\hline 330 & Cartones América & 380 & Papales Del Cauca & 430 & Caminos \\
\hline 331 & Cargraphics & 381 & Petrobras - Braspetro & 431 & Petrosantander \\
\hline 332 & Autogermana & 382 & Multidimensionales & 432 & Wyeth Consumer Healthcare \\
\hline 333 & Carbones De La Jagua & 383 & Merck & 433 & Pollos El Bucanero \\
\hline 334 & Coolechera & 384 & Productos Yupi & 434 & Clariant \\
\hline 335 & Construcciones El Cóndor & 385 & Campollo & 435 & Coca Cola Servicios \\
\hline 336 & Cementos Argos & 386 & Camargo Correa S.a. & 436 & Syngenta \\
\hline 337 & Bico International & 387 & Colmédica Emp & 437 & Asea Brown Boveri \\
\hline 338 & $3 \mathrm{~m}$ Colombia & 388 & Basf Química & 438 & Tintas \\
\hline 339 & Sea Tech & 389 & Metromed & 439 & Colombina Del Cauca \\
\hline 340 & Bonlam Andina & 390 & Kellogg De Colombia & 440 & Caracol Radio \\
\hline 341 & Alimentos Polar & 391 & Impsat & 441 & Motores Del Valle \\
\hline 342 & Termobarranquilla & 392 & U.Distrital Fco.José de Caldas & 442 & Cedelca \\
\hline 343 & Codiacero & 393 & Hospital Militar Central & 443 & Electrificadora Del Meta \\
\hline 344 & Cosméticos Votre Passion & 394 & Ci Dugotex & 444 & Ren Radio \\
\hline 345 & Mincivil & 395 & Casa Toro & 445 & Agrinal \\
\hline 346 & Almacenes Flamingo & 396 & Edatel & 446 & Comfenalco Valle Eps \\
\hline 347 & Diageo Colombia & 397 & Automotriz Interamericana & 447 & C.I. Emp Exp Colombiana de Café Ltd \\
\hline 348 & Ingenio La Cabaña & 398 & Cresta Roja & 448 & Superpolo \\
\hline 349 & Tecnosur & 399 & Ci Superalimentos & 449 & Productos Ramo \\
\hline 350 & Nabors Drilling & 400 & Valores Y Contratos & 450 & Ci Elite Flower \\
\hline
\end{tabular}




\begin{tabular}{|c|c|}
\hline No. & Empresa \\
\hline 451 & Arquitectos E Ingenieros Asociados \\
\hline 452 & Agro Grain S.a. \\
\hline 453 & Universidad De Pamplona \\
\hline 454 & Falabella De Colombia \\
\hline 455 & Portafolio De Inv. Suramericana \\
\hline 456 & Concentrados \\
\hline 457 & Agofer \\
\hline 458 & Agrícola Colombiana \\
\hline 459 & Servipetróleo \\
\hline 460 & Cooperan \\
\hline 461 & Omnilife \\
\hline 462 & Siderúrgica Del Occidente \\
\hline 463 & Cannon De Colombia \\
\hline 464 & Schneider Electric \\
\hline 465 & Ci Técnicas Baltime \\
\hline 466 & Comercializadora Giraldo Gómez \\
\hline 467 & Laumayer Exportadores De Café \\
\hline 468 & Luminex \\
\hline 469 & Tecnoglass \\
\hline 470 & Suramericana Arrend. Operativo \\
\hline 471 & Serdán \\
\hline 472 & Ci Mira \\
\hline 473 & Philips Colombiana \\
\hline 474 & Preparaciones De Belleza \\
\hline 475 & Ayura Motor \\
\hline 476 & Codiesel \\
\hline 477 & Productos Alimenticios Doria \\
\hline 478 & Coperacafé Manizalez \\
\hline 479 & Discristal \\
\hline 480 & Proalco \\
\hline 481 & Sidelpa \\
\hline 482 & Superpollo Paisa \\
\hline 483 & Mecánicos Asociados \\
\hline 484 & Telebucaramanga \\
\hline 485 & Independence Drilling S.a. \\
\hline 486 & Agro. El Búfalo \\
\hline 487 & Fundación Social \\
\hline 488 & Altipal Medellín \\
\hline 489 & Colwagen \\
\hline 490 & Casa Británica \\
\hline 491 & Grant Geophysical \\
\hline 492 & Industria Licorera De Caldas \\
\hline 493 & Proquinal \\
\hline 494 & Legis Legislación Económica \\
\hline 495 & Oracle Colombia \\
\hline 496 & Multienlace \\
\hline 497 & Textiles Miratex \\
\hline 498 & Alim. Concentrados Del Caribe \\
\hline 499 & Pvc Gerfor \\
\hline 500 & Ecoopsos Eps \\
\hline
\end{tabular}

